\title{
Prevalence and Clinical Characteristics of Hearing Loss Caused by MYH14 Variants
}

\author{
Ken Hiramatsu ${ }^{1}$, Shin-ya Nishio ${ }^{1,2}{ }^{\circledR}$, Shin-ichiro Kitajiri ${ }^{1}{ }^{1}$, Tomohiro Kitano ${ }^{1}$, Hideaki Moteki ${ }^{1}$, \\ Shin-ichi Usami ${ }^{1,2, * \mathbb{D}}$ and on behalf of the Deafness Gene Study Consortium ${ }^{\dagger}$ \\ 1 Department of Otorhinolaryngology, Shinshu University School of Medicine, 3-1-1 Asahi, Matsumoto, \\ Nagano 390-8621, Japan; matsuken@shinshu-u.ac.jp (K.H.); nishio@shinshu-u.ac.jp (S.-y.N.); \\ kitajiri@shinshu-u.ac.jp (S.-i.K.); tomokitano@shinshu-u.ac.jp (T.K.); moteki@shinshu-u.ac.jp (H.M.) \\ 2 Department of Hearing Implant Sciences, Shinshu University School of Medicine, 3-1-1 Asahi, Matsumoto, \\ Nagano 390-8621, Japan \\ * Correspondence: usami@shinshu-u.ac.jp; Tel.: +81-263-3802 \\ + Note: We thank some members of Deafness Gene Study Consortium
}

\section{check for} updates

Citation: Hiramatsu, K.; Nishio, S.-y.; Kitajiri, S.-i.; Kitano, T.; Moteki, H.;

Usami, S.-i.; on behalf of the Deafness Gene Study Consortium. Prevalence and Clinical Characteristics of Hearing Loss Caused by MYH14 Variants. Genes 2021, 12, 1623. https://doi.org/10.3390/ genes12101623

Academic Editor:

Regie Santos-Cortez

Received: 24 September 2021

Accepted: 11 October 2021

Published: 15 October 2021

Publisher's Note: MDPI stays neutral with regard to jurisdictional claims in published maps and institutional affiliations.

Copyright: (c) 2021 by the authors. Licensee MDPI, Basel, Switzerland. This article is an open access article distributed under the terms and conditions of the Creative Commons Attribution (CC BY) license (https:// creativecommons.org/licenses/by/ $4.0 /)$.

\begin{abstract}
Variants in MYH14 are reported to cause autosomal dominant nonsyndromic hereditary hearing loss (ADNSHL), with 34 variants reported to cause hearing loss in various ethnic groups. However, the available information on prevalence, as well as with regard to clinical features, remains fragmentary. In this study, genetic screening for MYH14 variants was carried out using a large series of Japanese hearing-loss patients to reveal more detailed information. Massively parallel DNA sequencing of 68 target candidate genes was applied in 8074 unrelated Japanese hearing-loss patients (including 1336 with ADNSHL) to identify genomic variations responsible for hearing loss. We identified 11 families with 10 variants. The prevalence was found to be $0.14 \%(11 / 8074)$ among all hearing-loss patients and $0.82 \%(11 / 1336)$ among ADNSHL patients. Nine of the eleven variants identified were novel. The patients typically showed late-onset hearing loss arising later than 20 years of age $(64.3 \%, 9 / 14)$ along with progressive $(92.3 \%, 12 / 13)$, moderate $(62.5 \%, 10 / 16)$, and flattype hearing loss $(68.8 \%, 11 / 16)$. We also confirmed progressive hearing loss in serial audiograms. The clinical information revealed by the present study will contribute to further diagnosis and management of MYH14-associated hearing loss.
\end{abstract}

Keywords: MYH14; nonsyndromic hearing loss; DFNA4; postlingual onset hearing loss; late-onset hearing loss; progressive hearing loss; massively parallel DNA sequencing

\section{Introduction}

Hearing loss (HL) is the most common sensory impairment. A wide variety of genes and variants have been reported as causative for $\mathrm{HL}$, and more than 120 genes have been reported to cause nonsyndromic HL [1]. The clinical features of HL, including age at onset, progression, severity, audiometric configuration, and effectiveness of interventions, differ among the various genes and variants [2]. Toward the more appropriate treatment of each HL patient, the identification of the causative variants by genetic testing and clarification of the clinical characteristics caused by each deafness gene variant are vital.

The inheritance patterns of hereditary HL include autosomal recessive, autosomal dominant, X-linked, and mitochondrial patterns. A majority of autosomal dominant nonsyndromic hereditary hearing loss (ADNSHL) is postlingual onset, progressive HL, which accounts for approximately $20 \%$ of nonsyndromic hereditary HL patients [2]. To date, 46 causative genes for ADNSHL have been identified [1].

MYH14 encodes nonmuscle myosin II C (NMIIC), a member of the myosin superfamily, and is a causative gene for ADNSHL (DFNA 4) [3]. It is widely expressed in the inner ear, including the organ of Corti. There have been reports of 34 MYH14 variants causing ADNSHL [4-24]. 
With regard to prevalence, four studies have investigated the prevalence of MYH14 variants in deafness cohorts from Europe, the USA, and China [4,8-10], but the information regarding clinical characteristics, including age at onset, progression, and audiometric configurations, remains fragmentary. In this study, we performed screening for MYH14 variants for a large number of Japanese HL patients $(n=8074$, including 1336 with ADNSNL), and examined the clinical features in detail.

\section{Materials and Methods}

\subsection{Subjects}

A total of 8074 Japanese HL patients were enrolled nationwide, as previously reported [25]. All patients showed sensorineural HL, with 1336 of them showing autosomal dominant (AD) inheritance patterns. This study was approved by the Shinshu University Ethical Committee, as well as the respective ethical committees of the other participating institutions, and was conducted in accordance with the Declaration of Helsinki. Informed consent was obtained from all patients (or from their next of kin, caretaker, or legal guardian in the cases of minors or children). Clinical information and peripheral blood samples were obtained from patients and all relatives from whom written informed consent was obtained.

\subsection{Variant Analysis}

Massively parallel DNA sequencing (MPS) analysis for 68 target deafness genes (Table S1) was performed for all patients. The detailed protocol was described elsewhere [25]. An Ion AmpliSeq Custom Panel (ThermoFisher Scientific, Waltham, MA, USA) was designed using an Ion AmpliSeq Designer, and the amplicon libraries were prepared using an Ion AmpliSeq library kit version 2.0 (ThermoFisher Scientific, Waltham, MA, USA). The emulsion PCR and MPS were performed using an Ion PGM, Ion Proton or IonS5 sequencer (ThermoFisher Scientific, Waltham, MA, USA), and the sequence data were mapped against the human genome sequence (build GRCh37/hg19).

The protein-affecting variants (including the missense, nonsense, insertion/deletion, and splicing variants) with an allele frequency of less than 1\% of the ExAC03 [26], ToMMo 3.5KJPN [27], and the 333 in-house Japanese normal hearing controls were selected. The annotation for each variant was analyzed by ANNOVAR software ver. 20191024 [28]. Functional in silico predictions were performed for missense variants by SIFT [29], PolyPhen2 [30], Mutation Taster [31], Mutation Assessor [32], FATHMM [33], and Combined Annotation Dependent Depletion (CADD) [34] software programs including in dbNSFP ver.3.5. The remaining MYH14 variants were confirmed by direct sequencing. Segregation analysis for family members was also performed by direct sequencing. The pathogenicity of the identified variants was evaluated using the American College of Medical Genetics (ACMG) standards and guidelines [35].

The variants classified as "Likely Pathogenic" or "Pathogenic" were considered to be causative variants. In addition, variants classified as being of "Uncertain significance" were also considered to be pathogenic if all three of the following conditions were satisfied: (1) no other candidate variants were identified in the other 67 genes; (2) the allele frequency was under 0.0001 in the control populations in ExAC03, gnomAD, ToMMo 3.5KJPN, and in-house controls; and (3) the CADD score was 20 or more.

\subsection{Clinical Evaluation}

Clinical information, including: (1) onset age; (2) progression of HL; (3) pedigree; (4) episodes or symptoms of vertigo; and (5) intervention for HL, was collected from a review of medical charts. Evaluation of HL was performed by pure-tone audiometry on patients aged 4 years or older, and the auditory steady-state response (ASSR) or play audiometry was performed for those who could not be evaluated by pure-tone audiometry. The pure-tone average (PTA) was calculated from the audiometric thresholds at four frequencies $(500,1000,2000$, and $4000 \mathrm{~Hz})$. The severity of HL was classified into 4 categories: mild (PTA $20-40 \mathrm{~dB})$, moderate $(41-70 \mathrm{~dB})$, severe (71-90 dB), and profound 
(>91 dB) [36]. The audiometric configurations were categorized into Flat, Low-frequency ascending, Mid-frequency U-shaped, High-frequency gently sloping, and High-frequency steeply sloping, as reported previously [36]. Caloric testing was performed for one of the two patients who complained of vestibular symptoms.

\section{Results}

Among the 1336 unrelated ADNSHL probands, we identified 11 probands (Table 1, Figure 1) carrying 10 possibly disease-causing MYH14 variants (Table 2). Among the 10 variants, 8 were novel. Therefore, the frequency of MYH14-related HL patients among Japanese ADNSHL patients was $0.82 \%(11 / 1336)$. Most of the MYH14 variants in previous reports were missense variants, with only two being nonsense variants (Table 3 ).

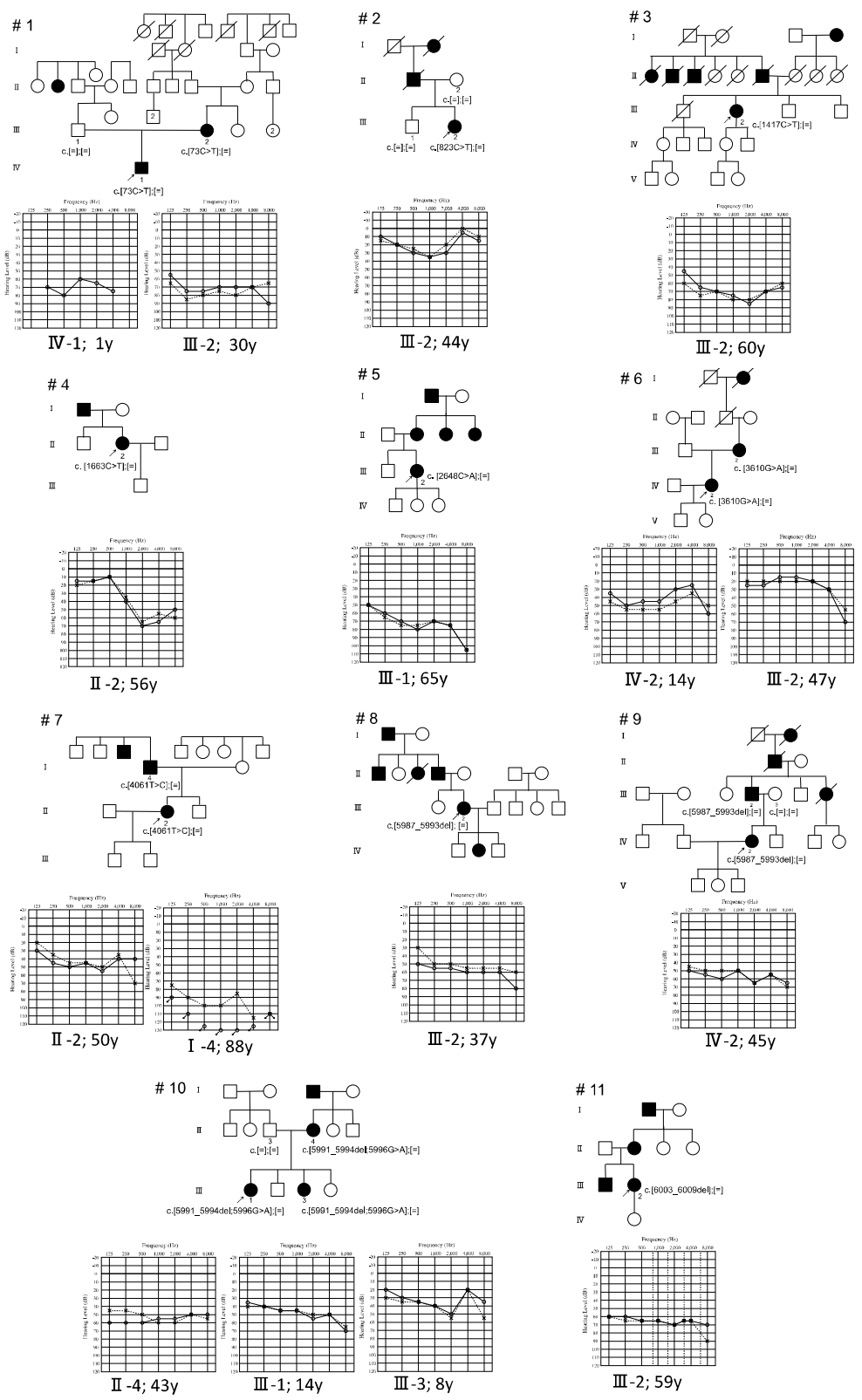

Figure 1. Pedigree, identified variants, and audiograms for the MYH14-associated hearing-loss patients identified in this study. Arrowheads indicate the proband for each family. Identified variants are indicated on the pedigree. Audiograms for the proband and other affected family members are shown with the age at hearing testing. 


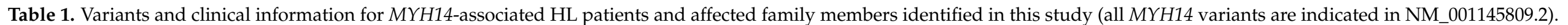

\begin{tabular}{|c|c|c|c|c|c|c|c|c|c|c|c|c|c|}
\hline $\begin{array}{l}\text { Nucleotide } \\
\text { Change }\end{array}$ & $\begin{array}{l}\text { Amino Acid } \\
\text { Change }\end{array}$ & Gender & Onset & $\begin{array}{l}\text { Progression } \\
\text { of HL }\end{array}$ & Age & $\begin{array}{l}\text { Audiometric } \\
\text { Configura- } \\
\text { tion }\end{array}$ & $\begin{array}{l}\text { PTA (Better- } \\
\text { Hearing Ear, } \\
\text { dB) }\end{array}$ & Severity & $\begin{array}{l}\text { Vestibular } \\
\text { Symptoms }\end{array}$ & Intervention & $\begin{array}{l}\text { Newborn } \\
\text { Hearing } \\
\text { Screening }\end{array}$ & Family No. & $\begin{array}{l}\text { Patient } \\
\text { No. }\end{array}$ \\
\hline c. $73 \mathrm{C}>\mathrm{T}$ & p.Q25X & $\mathrm{F}$ & $0 \mathrm{~s}$ & No & $30 \mathrm{Y}$ & Flat & 71.25 & Severe & No & HA & refer & 1 & III-2 \\
\hline c. $73 \mathrm{C}>\mathrm{T}$ & p.Q25X & M & 0 & $\mathrm{n} / \mathrm{a}$ & $1 \mathrm{Y}$ & Flat & 70 & Moderate & $\mathrm{n} / \mathrm{a}$ & $\mathrm{n} / \mathrm{a}$ & $\mathrm{n} / \mathrm{a}$ & 1 & IV-1 \\
\hline c. $823 \mathrm{C}>\mathrm{T}$ & p.R275C & $\mathrm{F}$ & $41 \mathrm{Y}$ & Yes & $44 \mathrm{Y}$ & U-shaped & 20 & Mild & No & $\mathrm{n} / \mathrm{a}$ & $\mathrm{n} / \mathrm{a}$ & 2 & III-2 \\
\hline c. $1417 \mathrm{C}>\mathrm{T}$ & p.R473C & $\mathrm{F}$ & $35 \mathrm{Y}$ & Yes & $60 Y$ & Flat & 75 & Severe & $\mathrm{n} / \mathrm{a}$ & $\mathrm{n} / \mathrm{a}$ & $\mathrm{n} / \mathrm{a}$ & 3 & III-2 \\
\hline c. $1663 \mathrm{C}>\mathrm{T}$ & p.P555S & $\mathrm{F}$ & $44 \mathrm{Y}$ & Yes & $56 \mathrm{Y}$ & $\begin{array}{l}\text { Steeply } \\
\text { sloping }\end{array}$ & 41.25 & Moderate & No & HA & $\mathrm{n} / \mathrm{a}$ & 4 & III-3 \\
\hline c. $2648 \mathrm{C}>\mathrm{A}$ & p.A883D & $\mathrm{F}$ & $44 \mathrm{Y}$ & $\mathrm{n} / \mathrm{a}$ & $65 \mathrm{Y}$ & $\begin{array}{l}\text { Gently } \\
\text { sloping }\end{array}$ & 73.75 & Moderate & Yes & $\mathrm{n} / \mathrm{a}$ & $\mathrm{n} / \mathrm{a}$ & 5 & III-1 \\
\hline c. $3610 \mathrm{G}>\mathrm{A}$ & p.E1204K & $\mathrm{F}$ & $12 \mathrm{Y}$ & Yes & $14 \mathrm{Y}$ & $\begin{array}{l}\text { frequency } \\
\text { ascending }\end{array}$ & 47.5 & Moderate & No & HA & $\mathrm{n} / \mathrm{a}$ & 6 & III-1 \\
\hline c. $3610 \mathrm{G}>\mathrm{A}$ & p.E1204K & $\mathrm{F}$ & $\mathrm{n} / \mathrm{a}$ & Yes & $47 \mathrm{Y}$ & Flat & 20 & Mild & No & $\mathrm{n} / \mathrm{a}$ & $\mathrm{n} / \mathrm{a}$ & 6 & III-3 \\
\hline c. $4061 \mathrm{~T}>\mathrm{C}$ & p.L1354P & M & $20 \mathrm{~s}$ & Yes & $83 \mathrm{Y}$ & Flat & 100 & Profound & $\mathrm{n} / \mathrm{a}$ & $\mathrm{n} / \mathrm{a}$ & $\mathrm{n} / \mathrm{a}$ & 7 & $\mathrm{I}-4$ \\
\hline c. $4061 \mathrm{~T}>\mathrm{C}$ & p.L1354P & $\mathrm{F}$ & $30 \mathrm{~s}$ & Yes & $50 \mathrm{Y}$ & Flat & 43.75 & Moderate & No & HA & $\mathrm{n} / \mathrm{a}$ & 7 & II-2 \\
\hline c.5987_5993del & p.R1997Afs * & $\mathrm{F}$ & $20 Y$ & Yes & $37 \mathrm{Y}$ & Flat & 53.75 & Moderate & Yes & HA & $\mathrm{n} / \mathrm{a}$ & 8 & III-2 \\
\hline c.5987_5993del & p.R1997Afs * & $\mathrm{F}$ & $20 \mathrm{~s}$ & Yes & $45 \mathrm{Y}$ & Flat & 55 & Moderate & No & HA & $\mathrm{n} / \mathrm{a}$ & 9 & IV-2 \\
\hline c.5987_5993del & p.R1997Afs * & M & $\mathrm{n} / \mathrm{a}$ & $\mathrm{n} / \mathrm{a}$ & $\mathrm{n} / \mathrm{a}$ & $\mathrm{n} / \mathrm{a}$ & $\mathrm{n} / \mathrm{a}$ & $\mathrm{n} / \mathrm{a}$ & $\mathrm{n} / \mathrm{a}$ & $\mathrm{n} / \mathrm{a}$ & $\mathrm{n} / \mathrm{a}$ & 9 & III-2 \\
\hline $\begin{array}{c}\text { c.[5991_5994del; } \\
\text { 5996G > A] }\end{array}$ & $\begin{array}{l}\text { p.[V1998Afs *; } \\
\text { R1999H] }\end{array}$ & F & $\mathrm{n} / \mathrm{a}$ & $\mathrm{n} / \mathrm{a}$ & $43 Y$ & Flat & 55 & Moderate & $\mathrm{n} / \mathrm{a}$ & $\mathrm{n} / \mathrm{a}$ & $\mathrm{n} / \mathrm{a}$ & 10 & III-4 \\
\hline $\begin{array}{c}\text { c. [5991_5994del; } \\
\text { 5996G > A] }\end{array}$ & $\begin{array}{l}\text { p.[V1998Afs *; } \\
\text { R1999H] }\end{array}$ & F & $3 Y$ & Yes & $8 \mathrm{Y}$ & U-shaped & 29 & Mild & No & HA & pass & 10 & III-3 \\
\hline c.6003_6009del & p.F2002X & $\mathrm{F}$ & $41 \mathrm{Y}$ & Yes & $59 \mathrm{Y}$ & Flat & 66.25 & Moderate & No & $\mathrm{n} / \mathrm{a}$ & $\mathrm{n} / \mathrm{a}$ & 11 & III-2 \\
\hline
\end{tabular}

${ }^{*}$ indicate the stop codon as in standrd HGVS nomenclature. n/a, not available; HA, hearing aids; CI, cochlear implant. Family No. and Patient No. indicate the patient number in Figure 1. 
Table 2. Identified MYH14 variants and in silico predication score (all MYH14 variants are indicated in NM_001145809.2).

\begin{tabular}{|c|c|c|c|c|c|c|c|c|c|c|c|c|c|c|}
\hline $\begin{array}{l}\text { Nucleotide } \\
\text { Change }\end{array}$ & Exon & $\begin{array}{l}\text { Amino Acid } \\
\text { Change }\end{array}$ & Domain & SIFT & PP2 & $\begin{array}{l}\text { Mut } \\
\text { Taster }\end{array}$ & $\begin{array}{c}\text { Mut } \\
\text { Assessor }\end{array}$ & Revel & $\begin{array}{l}\text { CADD } \\
\text { Phred }\end{array}$ & $\begin{array}{c}\text { Allele } \\
\text { Frequency } \\
\text { in In-house } \\
\text { Controls }\end{array}$ & $\begin{array}{l}\text { MAF in } \\
\text { ExAC03 }\end{array}$ & $\begin{array}{l}\text { MAF in } \\
\text { ToMMo } \\
(4.7 k J P N)\end{array}$ & $\begin{array}{l}\text { ACMG } \\
\text { Criteria }\end{array}$ & Reference \\
\hline c. $73 \mathrm{C}>\mathrm{T}$ & 2 & p.Q25X & $\begin{array}{c}\text { Myosin, } \\
\text { N-terminal }\end{array}$ & & & A & & & 36 & 0 & 0 & 0 & $\begin{array}{c}\text { Likely } \\
\text { Pathogenic }\end{array}$ & $\begin{array}{c}\text { Donaudy et al., } \\
2004 \text { [3] }\end{array}$ \\
\hline c. $823 \mathrm{C}>\mathrm{T}$ & 8 & p.R275C & $\begin{array}{l}\text { Myosin head, } \\
\text { motor } \\
\text { domain }\end{array}$ & $\mathrm{D}$ & $\mathrm{D}$ & $\mathrm{D}$ & $\mathrm{D}$ & 0.648 & 34 & 0 & 0.000008252 & 0 & $\begin{array}{l}\text { Uncertain } \\
\text { Significance }\end{array}$ & $\begin{array}{c}\text { Iwasa et al., } \\
2016 \text { [6] }\end{array}$ \\
\hline c. $1417 \mathrm{C}>\mathrm{T}$ & 13 & p.R473C & $\begin{array}{l}\text { Myosin head, } \\
\text { motor } \\
\text { domain }\end{array}$ & $\mathrm{D}$ & $\mathrm{P}$ & $\mathrm{D}$ & M & 0.648 & 34 & 0 & $\begin{array}{c}2.81 \times \\
10^{-5}\end{array}$ & 0 & $\begin{array}{l}\text { Uncertain } \\
\text { Significance }\end{array}$ & This study \\
\hline c. $1663 \mathrm{C}>\mathrm{T}$ & 15 & p.P555S & $\begin{array}{l}\text { Myosin head, } \\
\text { motor } \\
\text { domain }\end{array}$ & $\mathrm{D}$ & $\mathrm{D}$ & $\mathrm{D}$ & M & 0.701 & 26.1 & 0 & 0 & 0 & $\begin{array}{l}\text { Uncertain } \\
\text { Significance }\end{array}$ & This study \\
\hline c. $2648 \mathrm{C}>\mathrm{A}$ & 22 & p.A883D & Myosin tail & $\mathrm{D}$ & $\mathrm{D}$ & $\mathrm{D}$ & M & 0.692 & 28 & 0 & 0 & 0 & $\begin{array}{l}\text { Uncertain } \\
\text { Significance }\end{array}$ & This study \\
\hline c. $3610 \mathrm{G}>\mathrm{A}$ & 28 & p.E1204K & Myosin tail & $\mathrm{T}$ & B & $\mathrm{D}$ & $\mathrm{L}$ & 0.678 & 24.7 & 0 & $\begin{array}{c}2.29 \times \\
10^{-5}\end{array}$ & 0 & $\begin{array}{l}\text { Uncertain } \\
\text { Significance }\end{array}$ & This study \\
\hline c. $4061 \mathrm{~T}>\mathrm{C}$ & 31 & p.L1354P & Myosin tail & $\mathrm{D}$ & $\mathrm{D}$ & $\mathrm{D}$ & M & 0.813 & 28.4 & 0 & 0 & 0 & $\begin{array}{l}\text { Uncertain } \\
\text { Significance }\end{array}$ & This study \\
\hline c.5987_5993del & & p.R1997Afs & & & & & & & & 0 & 0 & 0 & $\begin{array}{l}\text { Uncertain } \\
\text { Significance }\end{array}$ & This study \\
\hline $\begin{array}{l}\text { c. [5991_5994del; } \\
\text { 5996G > A] }\end{array}$ & 43 & $\begin{array}{l}\text { p.[V1998Afs; } \\
\text { R1999H] }\end{array}$ & & $\mathrm{D}$ & $\mathrm{P}$ & $\mathrm{N}$ & $\mathrm{L}$ & 0.251 & 24.1 & 0 & $\begin{array}{c}3.35 \times \\
10^{-5}\end{array}$ & 0 & $\begin{array}{l}\text { Uncertain } \\
\text { Significance }\end{array}$ & This study \\
\hline c.6003_6009del & 43 & p.F2002X & & & & & & & & 0 & 0 & 0 & $\begin{array}{l}\text { Uncertain } \\
\text { Significance }\end{array}$ & This study \\
\hline
\end{tabular}

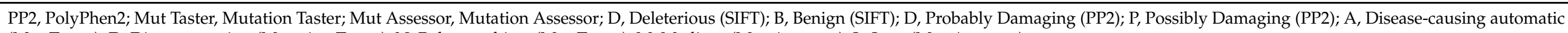
(Mut Taster); D, Disease-causing (Mutation Taster); N, Polymorphism (Mut Taster); M, Medium (Mut Assessor); L, Low (Mut Assessor). 
Table 3. MYH14 variants and clinical information in previous reports (all MYH14 variants are indicated in NM_001145809.2).

\begin{tabular}{|c|c|c|c|c|c|c|c|c|c|c|c|c|}
\hline $\begin{array}{l}\text { Nucleotide } \\
\text { Change }\end{array}$ & $\begin{array}{l}\text { Amino Acid } \\
\text { Change }\end{array}$ & Gender & Onset & $\begin{array}{l}\text { Progression of } \\
\text { HL }\end{array}$ & Age & $\begin{array}{c}\text { Audiometric } \\
\text { Configuration }\end{array}$ & $\begin{array}{l}\text { PTA (Better- } \\
\text { Hearing } \\
\text { Ear) }\end{array}$ & Severity & $\begin{array}{l}\text { Vestibular } \\
\text { Symptoms }\end{array}$ & Intervention & $\begin{array}{l}\text { Newborn } \\
\text { Hearing } \\
\text { Screening }\end{array}$ & Reference \\
\hline c. $20 \mathrm{C}>\mathrm{A}$ & p.S7X & $\mathrm{n} / \mathrm{a}$ & 10 or 20 & Yes & $\mathrm{n} / \mathrm{a}$ & $\mathrm{n} / \mathrm{a}$ & $\mathrm{n} / \mathrm{a}$ & $\begin{array}{l}\text { Severe to } \\
\text { profound in } 40 \\
\text { years }\end{array}$ & $\mathrm{n} / \mathrm{a}$ & $\mathrm{n} / \mathrm{a}$ & $\mathrm{n} / \mathrm{a}$ & Donaudy et al., 2004 [3] \\
\hline \multirow{3}{*}{ c. $73 \mathrm{C}>\mathrm{T}$} & \multirow{3}{*}{ p.Q25X } & $\mathrm{F}$ & $0 \mathrm{Y}$ & Yes & $7 \mathrm{M}$ & $\mathrm{n} / \mathrm{a}$ & $\mathrm{n} / \mathrm{a}$ & $\mathrm{n} / \mathrm{a}$ & $\mathrm{n} / \mathrm{a}$ & $\mathrm{n} / \mathrm{a}$ & $\mathrm{n} / \mathrm{a}$ & \multirow{3}{*}{ Kim et al., 2017 [4] } \\
\hline & & $\mathrm{F}$ & $\mathrm{n} / \mathrm{a}$ & $\mathrm{n} / \mathrm{a}$ & $33 \mathrm{Y}$ & Flat & 82.5 & Severe & $\mathrm{n} / \mathrm{a}$ & $\mathrm{n} / \mathrm{a}$ & $\mathrm{n} / \mathrm{a}$ & \\
\hline & & $\mathrm{n} / \mathrm{a}$ & $\mathrm{n} / \mathrm{a}$ & $\mathrm{n} / \mathrm{a}$ & $28 \mathrm{Y}$ & Flat & 53.8 & Moderate & $\mathrm{n} / \mathrm{a}$ & $\mathrm{n} / \mathrm{a}$ & $\mathrm{n} / \mathrm{a}$ & \\
\hline \multirow{3}{*}{ c. $359 \mathrm{C}>\mathrm{T}$} & \multirow{3}{*}{ p.S120L } & $\mathrm{n} / \mathrm{a}$ & $\mathrm{n} / \mathrm{a}$ & $\mathrm{n} / \mathrm{a}$ & $33 \mathrm{Y}$ & Flat & 65 & Moderate & $\mathrm{n} / \mathrm{a}$ & $\mathrm{n} / \mathrm{a}$ & $\mathrm{n} / \mathrm{a}$ & \multirow{3}{*}{ Yang et al., 2005 [5] } \\
\hline & & $\mathrm{n} / \mathrm{a}$ & $\mathrm{n} / \mathrm{a}$ & $\mathrm{n} / \mathrm{a}$ & $35 \mathrm{Y}$ & Flat & 76.3 & Moderate & $\mathrm{n} / \mathrm{a}$ & $\mathrm{n} / \mathrm{a}$ & $\mathrm{n} / \mathrm{a}$ & \\
\hline & & $\mathrm{n} / \mathrm{a}$ & $\mathrm{n} / \mathrm{a}$ & $\mathrm{n} / \mathrm{a}$ & $63 \mathrm{Y}$ & Flat & 72.5 & Moderate & $\mathrm{n} / \mathrm{a}$ & $\mathrm{n} / \mathrm{a}$ & $\mathrm{n} / \mathrm{a}$ & \\
\hline \multirow{4}{*}{ c. $541 \mathrm{G}>\mathrm{A}$} & p.A176T & $\mathrm{n} / \mathrm{a}$ & $\mathrm{n} / \mathrm{a}$ & $\mathrm{n} / \mathrm{a}$ & $\mathrm{n} / \mathrm{a}$ & $\mathrm{n} / \mathrm{a}$ & $\mathrm{n} / \mathrm{a}$ & $\mathrm{n} / \mathrm{a}$ & $\mathrm{n} / \mathrm{a}$ & $\mathrm{n} / \mathrm{a}$ & $\mathrm{n} / \mathrm{a}$ & Chen et al., 2016 [9] \\
\hline & \multirow{3}{*}{ p.A181T } & $\mathrm{F}$ & $\begin{array}{l}\text { The first } \\
\text { decade }\end{array}$ & $\mathrm{n} / \mathrm{a}$ & $\mathrm{n} / \mathrm{a}$ & U-sharped & 73.75 & Moderate & $\mathrm{n} / \mathrm{a}$ & $\mathrm{n} / \mathrm{a}$ & $\mathrm{n} / \mathrm{a}$ & \multirow{3}{*}{ Qing et al., 2014 [10] } \\
\hline & & $\mathrm{M}$ & $\begin{array}{l}\text { The first } \\
\text { decade }\end{array}$ & $\mathrm{n} / \mathrm{a}$ & $\mathrm{n} / \mathrm{a}$ & Flat & 71.3 & Moderate & $\mathrm{n} / \mathrm{a}$ & $\mathrm{n} / \mathrm{a}$ & $\mathrm{n} / \mathrm{a}$ & \\
\hline & & $\mathrm{F}$ & $\begin{array}{l}\text { The first } \\
\text { decade }\end{array}$ & $\mathrm{n} / \mathrm{a}$ & $\mathrm{n} / \mathrm{a}$ & U-sharped & $\mathrm{n} / \mathrm{a}$ & Severe & $\mathrm{n} / \mathrm{a}$ & $\mathrm{n} / \mathrm{a}$ & $\mathrm{n} / \mathrm{a}$ & \\
\hline c. $572 \mathrm{~A}>\mathrm{G}$ & p.D191G & M & $\begin{array}{c}\text { Congenital } \\
\text { or prelingual }\end{array}$ & No? & $5 \mathrm{Y}$ & Flat & 71.3 & Moderate & $\mathrm{n} / \mathrm{a}$ & $\mathrm{n} / \mathrm{a}$ & $\mathrm{n} / \mathrm{a}$ & Kim et al., 2017 [4] \\
\hline c. $823 \mathrm{C}>\mathrm{T}$ & p.R275C & $\mathrm{F}$ & $41 Y$ & Yes & $44 \mathrm{Y}$ & U-shaped & 20 & Mild & No & $\mathrm{n} / \mathrm{a}$ & $\mathrm{n} / \mathrm{a}$ & Iwasa et al., 2016 [6] \\
\hline c. $1049 \mathrm{G}>\mathrm{A}$ & p.R350Q & $\mathrm{n} / \mathrm{a}$ & $\mathrm{n} / \mathrm{a}$ & $\mathrm{n} / \mathrm{a}$ & $\mathrm{n} / \mathrm{a}$ & $\mathrm{n} / \mathrm{a}$ & $\mathrm{n} / \mathrm{a}$ & $\mathrm{n} / \mathrm{a}$ & $\mathrm{n} / \mathrm{a}$ & $\mathrm{n} / \mathrm{a}$ & $\mathrm{n} / \mathrm{a}$ & Iwasa et al., 2016 [6] \\
\hline \multirow{2}{*}{ c. $1067 \mathrm{C}>\mathrm{T}$} & p.T356M & $\mathrm{n} / \mathrm{a}$ & $\mathrm{n} / \mathrm{a}$ & $\mathrm{n} / \mathrm{a}$ & $\mathrm{n} / \mathrm{a}$ & $\mathrm{n} / \mathrm{a}$ & $\mathrm{n} / \mathrm{a}$ & $\mathrm{n} / \mathrm{a}$ & $\mathrm{n} / \mathrm{a}$ & $\mathrm{n} / \mathrm{a}$ & $\mathrm{n} / \mathrm{a}$ & Sommen et al., 2016 [11] \\
\hline & & $\mathrm{n} / \mathrm{a}$ & $\mathrm{n} / \mathrm{a}$ & $\mathrm{n} / \mathrm{a}$ & $9 \mathrm{Y}$ & $\mathrm{n} / \mathrm{a}$ & $\mathrm{n} / \mathrm{a}$ & Moderate & No & $\mathrm{n} / \mathrm{a}$ & $\mathrm{n} / \mathrm{a}$ & Donaudy., 2004 [3] \\
\hline c. $1360 \mathrm{G}>\mathrm{A}$ & p.A454T & $\mathrm{n} / \mathrm{a}$ & $\mathrm{n} / \mathrm{a}$ & $\mathrm{n} / \mathrm{a}$ & $\mathrm{n} / \mathrm{a}$ & $\mathrm{n} / \mathrm{a}$ & $\mathrm{n} / \mathrm{a}$ & $\mathrm{n} / \mathrm{a}$ & $\mathrm{n} / \mathrm{a}$ & $\mathrm{n} / \mathrm{a}$ & $\mathrm{n} / \mathrm{a}$ & Chen et al., 2016 [9] \\
\hline c. $1427 \mathrm{G}>\mathrm{A}$ & p.R476H & $\mathrm{n} / \mathrm{a}$ & $\mathrm{n} / \mathrm{a}$ & $\mathrm{n} / \mathrm{a}$ & $\mathrm{n} / \mathrm{a}$ & $\mathrm{n} / \mathrm{a}$ & $\mathrm{n} / \mathrm{a}$ & $\mathrm{n} / \mathrm{a}$ & $\mathrm{n} / \mathrm{a}$ & $\mathrm{n} / \mathrm{a}$ & $\mathrm{n} / \mathrm{a}$ & Sloan-Heggen et al., 2016 [8] \\
\hline c. $1609 \mathrm{G}>\mathrm{A}$ & p.D537N & $\mathrm{F}$ & $\mathrm{n} / \mathrm{a}$ & Yes & $8 \mathrm{Y}$ & Flat & 40 & Moderate & $\mathrm{n} / \mathrm{a}$ & $\mathrm{n} / \mathrm{a}$ & $\mathrm{n} / \mathrm{a}$ & Kim et al., 2015 [14] \\
\hline c. $1625 \mathrm{~T}>\mathrm{G}$ & p.L542R & $\mathrm{n} / \mathrm{a}$ & $\mathrm{n} / \mathrm{a}$ & $\mathrm{n} / \mathrm{a}$ & $\mathrm{n} / \mathrm{a}$ & $\mathrm{n} / \mathrm{a}$ & $\mathrm{n} / \mathrm{a}$ & $\mathrm{n} / \mathrm{a}$ & $\mathrm{n} / \mathrm{a}$ & $\mathrm{n} / \mathrm{a}$ & $\mathrm{n} / \mathrm{a}$ & Sloan-Heggen et al., 2016 [8] \\
\hline c. $1919 \mathrm{G}>\mathrm{A}$ & p.R640Q & $\mathrm{n} / \mathrm{a}$ & $\mathrm{n} / \mathrm{a}$ & $\mathrm{n} / \mathrm{a}$ & $\mathrm{n} / \mathrm{a}$ & $\mathrm{n} / \mathrm{a}$ & $\mathrm{n} / \mathrm{a}$ & $\mathrm{n} / \mathrm{a}$ & $\mathrm{n} / \mathrm{a}$ & $\mathrm{n} / \mathrm{a}$ & $\mathrm{n} / \mathrm{a}$ & Shearer et al., 2013 [7] \\
\hline c. $2089 \mathrm{G}>\mathrm{A}$ & p.G697S & $\mathrm{n} / \mathrm{a}$ & $\mathrm{n} / \mathrm{a}$ & $\mathrm{n} / \mathrm{a}$ & $\mathrm{n} / \mathrm{a}$ & $\mathrm{n} / \mathrm{a}$ & $\mathrm{n} / \mathrm{a}$ & $\mathrm{n} / \mathrm{a}$ & $\mathrm{n} / \mathrm{a}$ & $\mathrm{n} / \mathrm{a}$ & $\mathrm{n} / \mathrm{a}$ & Iwasa et al., 2016 [6] \\
\hline \multirow{2}{*}{ c. $2203 C>G$} & p.R735C & M & $\mathrm{n} / \mathrm{a}$ & $\mathrm{n} / \mathrm{a}$ & $\mathrm{n} / \mathrm{a}$ & Flat & 115 & Severe & $\mathrm{n} / \mathrm{a}$ & CI & $\mathrm{n} / \mathrm{a}$ & \\
\hline & p.ersoce & $\mathrm{M}$ & $\mathrm{n} / \mathrm{a}$ & $\mathrm{n} / \mathrm{a}$ & $\mathrm{n} / \mathrm{a}$ & $\mathrm{n} / \mathrm{a}$ & $\mathrm{n} / \mathrm{a}$ & $\mathrm{n} / \mathrm{a}$ & $\mathrm{n} / \mathrm{a}$ & $\mathrm{n} / \mathrm{a}$ & $\mathrm{n} / \mathrm{a}$ & Liu et al., 2019 [15] \\
\hline
\end{tabular}


Table 3. Cont.

\begin{tabular}{|c|c|c|c|c|c|c|c|c|c|c|c|c|}
\hline $\begin{array}{l}\text { Nucleotide } \\
\text { Change }\end{array}$ & $\begin{array}{l}\text { Amino Acid } \\
\text { Change }\end{array}$ & Gender & Onset & $\begin{array}{l}\text { Progression of } \\
\text { HL }\end{array}$ & Age & $\begin{array}{l}\text { Audiometric } \\
\text { Configuration }\end{array}$ & $\begin{array}{l}\text { PTA (Better- } \\
\text { Hearing } \\
\text { Ear) }\end{array}$ & Severity & $\begin{array}{l}\text { Vestibular } \\
\text { Symptoms }\end{array}$ & Intervention & $\begin{array}{c}\text { Newborn } \\
\text { Hearing } \\
\text { Screening }\end{array}$ & Reference \\
\hline c. $2299 \mathrm{C}>\mathrm{A}$ & p.R767S & $\mathrm{n} / \mathrm{a}$ & $\mathrm{n} / \mathrm{a}$ & Yes & $\mathrm{n} / \mathrm{a}$ & $\mathrm{n} / \mathrm{a}$ & $\mathrm{n} / \mathrm{a}$ & $\begin{array}{l}\text { Mild to } \\
\text { moderate }\end{array}$ & No & $\mathrm{n} / \mathrm{a}$ & $\mathrm{n} / \mathrm{a}$ & Donaudy et al., 2004 [3] \\
\hline $\begin{array}{l}\text { c. } 2621 \mathrm{~T}>\mathrm{C} \\
\text { c. } 2692 \mathrm{~A}>\mathrm{C}\end{array}$ & $\begin{array}{l}\text { p.L874P } \\
\text { p.K898Q }\end{array}$ & $\begin{array}{l}\mathrm{n} / \mathrm{a} \\
\mathrm{n} / \mathrm{a}\end{array}$ & $\begin{array}{l}\mathrm{n} / \mathrm{a} \\
\mathrm{n} / \mathrm{a}\end{array}$ & $\begin{array}{l}\mathrm{n} / \mathrm{a} \\
\mathrm{n} / \mathrm{a}\end{array}$ & $\begin{array}{l}\mathrm{n} / \mathrm{a} \\
\mathrm{n} / \mathrm{a}\end{array}$ & $\begin{array}{l}\mathrm{n} / \mathrm{a} \\
\mathrm{n} / \mathrm{a}\end{array}$ & $\begin{array}{l}\mathrm{n} / \mathrm{a} \\
\mathrm{n} / \mathrm{a}\end{array}$ & $\begin{array}{l}\text { moderate } \\
\text { n/a } \\
n / a\end{array}$ & $\begin{array}{l}\mathrm{n} / \mathrm{a} \\
\mathrm{n} / \mathrm{a}\end{array}$ & $\begin{array}{l}\mathrm{n} / \mathrm{a} \\
\mathrm{n} / \mathrm{a}\end{array}$ & $\begin{array}{l}\mathrm{n} / \mathrm{a} \\
\mathrm{n} / \mathrm{a}\end{array}$ & $\begin{array}{l}\text { Chen et al., } 2016 \text { [9] } \\
\text { Miyagawa et al., } 2013 \text { [16] }\end{array}$ \\
\hline \multirow[t]{2}{*}{ c. $2717 \mathrm{C}>\mathrm{T}$} & \multirow[t]{2}{*}{ p.T906M } & F & $\begin{array}{l}\text { The first } \\
\text { decade }\end{array}$ & $\mathrm{n} / \mathrm{a}$ & $\mathrm{n} / \mathrm{a}$ & U-shaped & 83 & Severe & $\mathrm{n} / \mathrm{a}$ & $\mathrm{n} / \mathrm{a}$ & $\mathrm{n} / \mathrm{a}$ & Qing et al., 2014 [10] \\
\hline & & $\mathrm{F}$ & $\begin{array}{l}\text { The first } \\
\text { decade }\end{array}$ & $\mathrm{n} / \mathrm{a}$ & $\mathrm{n} / \mathrm{a}$ & U-shaped & 80 & Severe & $\mathrm{n} / \mathrm{a}$ & $\mathrm{n} / \mathrm{a}$ & $\mathrm{n} / \mathrm{a}$ & \\
\hline \multirow[t]{2}{*}{ c. $2921 \mathrm{G}>\mathrm{A}$} & \multirow[t]{2}{*}{ p.R974H } & $\begin{array}{c}\mathrm{n} / \mathrm{a} \\
\mathrm{M}\end{array}$ & $\begin{array}{l}\mathrm{n} / \mathrm{a} \\
\mathrm{n} / \mathrm{a}\end{array}$ & $\begin{array}{l}\mathrm{n} / \mathrm{a} \\
\mathrm{n} / \mathrm{a}\end{array}$ & $\begin{array}{l}\mathrm{n} / \mathrm{a} \\
52 \mathrm{Y}\end{array}$ & $\begin{array}{l}\mathrm{n} / \mathrm{a} \\
\mathrm{n} / \mathrm{a}\end{array}$ & $\begin{array}{l}\mathrm{n} / \mathrm{a} \\
\mathrm{n} / \mathrm{a}\end{array}$ & $\begin{array}{l}\mathrm{n} / \mathrm{a} \\
\mathrm{n} / \mathrm{a}\end{array}$ & $\begin{array}{l}\mathrm{n} / \mathrm{a} \\
\mathrm{n} / \mathrm{a}\end{array}$ & $\mathrm{n} / \mathrm{a}$ & $\begin{array}{l}\mathrm{n} / \mathrm{a} \\
\mathrm{n} / \mathrm{a}\end{array}$ & Sloan-Heggen et al., 2016 [8] \\
\hline & & $\mathrm{M}$ & $\mathrm{n} / \mathrm{a}$ & $\mathrm{n} / \mathrm{a}$ & $48 \mathrm{Y}$ & $\mathrm{n} / \mathrm{a}$ & $\mathrm{n} / \mathrm{a}$ & $\mathrm{n} / \mathrm{a}$ & $\mathrm{n} / \mathrm{a}$ & $\mathrm{n} / \mathrm{a}$ & $\mathrm{n} / \mathrm{a}$ & \\
\hline \multirow{3}{*}{ c. $2921 \mathrm{G}>\mathrm{T}$} & \multirow[t]{3}{*}{ p.R974L } & $\mathrm{F}$ & $\mathrm{n} / \mathrm{a}$ & $\mathrm{n} / \mathrm{a}$ & $45 \mathrm{Y}$ & $\mathrm{n} / \mathrm{a}$ & $\mathrm{n} / \mathrm{a}$ & $\mathrm{n} / \mathrm{a}$ & $\mathrm{n} / \mathrm{a}$ & $\mathrm{n} / \mathrm{a}$ & $\mathrm{n} / \mathrm{a}$ & Choi et al., 2011 [17] \\
\hline & & $\mathrm{F}$ & $\mathrm{n} / \mathrm{a}$ & $\mathrm{n} / \mathrm{a}$ & $41 \mathrm{Y}$ & $\mathrm{n} / \mathrm{a}$ & $\mathrm{n} / \mathrm{a}$ & $\mathrm{n} / \mathrm{a}$ & $\mathrm{n} / \mathrm{a}$ & $\mathrm{n} / \mathrm{a}$ & $\mathrm{n} / \mathrm{a}$ & \\
\hline & & $\mathrm{M}$ & $\mathrm{n} / \mathrm{a}$ & $\mathrm{n} / \mathrm{a}$ & $15 \mathrm{Y}$ & $\mathrm{n} / \mathrm{a}$ & $\mathrm{n} / \mathrm{a}$ & $\mathrm{n} / \mathrm{a}$ & $\mathrm{n} / \mathrm{a}$ & $\mathrm{n} / \mathrm{a}$ & $\mathrm{n} / \mathrm{a}$ & \\
\hline \multirow[t]{2}{*}{ c. $2921 \mathrm{G}>\mathrm{T}$} & \multirow[t]{2}{*}{ p.R974L } & $\mathrm{n} / \mathrm{a}$ & $20 \mathrm{Y}$ & Yes & $\mathrm{n} / \mathrm{a}$ & $\mathrm{n} / \mathrm{a}$ & $\mathrm{n} / \mathrm{a}$ & $\mathrm{n} / \mathrm{a}$ & $\mathrm{n} / \mathrm{a}$ & $\mathrm{n} / \mathrm{a}$ & $\mathrm{n} / \mathrm{a}$ & Iyadurai et al., 2017 [20] \\
\hline & & $\mathrm{F}$ & $\mathrm{n} / \mathrm{a}$ & $\mathrm{n} / \mathrm{a}$ & $\mathrm{n} / \mathrm{a}$ & $\mathrm{n} / \mathrm{a}$ & $\mathrm{n} / \mathrm{a}$ & $\mathrm{n} / \mathrm{a}$ & $\mathrm{n} / \mathrm{a}$ & $\mathrm{n} / \mathrm{a}$ & $\mathrm{n} / \mathrm{a}$ & \\
\hline \multirow[t]{2}{*}{ c. $2921 \mathrm{G}>\mathrm{A}$} & \multirow[t]{2}{*}{ p.R974L } & $\begin{array}{l}\mathrm{F} \\
\mathrm{M}\end{array}$ & $\begin{array}{l}\mathrm{n} / \mathrm{a} \\
\mathrm{n} / \mathrm{a}\end{array}$ & $\begin{array}{l}\mathrm{n} / \mathrm{a} \\
\mathrm{n} / \mathrm{a}\end{array}$ & $\begin{array}{l}58 \mathrm{Y} \\
23 \mathrm{Y}\end{array}$ & $\begin{array}{l}\mathrm{n} / \mathrm{a} \\
\mathrm{n} / \mathrm{a}\end{array}$ & $\begin{array}{l}\mathrm{n} / \mathrm{a} \\
\mathrm{n} / \mathrm{a}\end{array}$ & $\begin{array}{l}\mathrm{n} / \mathrm{a} \\
\mathrm{n} / \mathrm{a}\end{array}$ & $\begin{array}{l}\mathrm{n} / \mathrm{a} \\
\mathrm{n} / \mathrm{a}\end{array}$ & $\begin{array}{l}\mathrm{n} / \mathrm{a} \\
\mathrm{n} / \mathrm{a}\end{array}$ & $\begin{array}{l}\mathrm{n} / \mathrm{a} \\
\mathrm{n} / \mathrm{a}\end{array}$ & Almutawa et al., 2019 [21] \\
\hline & & $\mathrm{F}$ & $\mathrm{n} / \mathrm{a}$ & $\mathrm{n} / \mathrm{a}$ & $24 \mathrm{Y}$ & $\mathrm{n} / \mathrm{a}$ & $\mathrm{n} / \mathrm{a}$ & $\mathrm{n} / \mathrm{a}$ & $\mathrm{n} / \mathrm{a}$ & $\mathrm{n} / \mathrm{a}$ & $\mathrm{n} / \mathrm{a}$ & \\
\hline c. $3049 \mathrm{C}>\mathrm{T}$ & p.L1017F & M & $\mathrm{n} / \mathrm{a}$ & $\mathrm{n} / \mathrm{a}$ & $\mathrm{n} / \mathrm{a}$ & $\mathrm{n} / \mathrm{a}$ & $\mathrm{n} / \mathrm{a}$ & $\begin{array}{l}\text { Mild to } \\
\text { moderate }\end{array}$ & No & $\mathrm{n} / \mathrm{a}$ & $\mathrm{n} / \mathrm{a}$ & Donaudy et al., 2004 [3] \\
\hline c. $3877 \mathrm{G}>\mathrm{C}$ & p.E1293Q & $\mathrm{n} / \mathrm{a}$ & $\mathrm{n} / \mathrm{a}$ & $\mathrm{n} / \mathrm{a}$ & $\mathrm{n} / \mathrm{a}$ & $\mathrm{n} / \mathrm{a}$ & $\mathrm{n} / \mathrm{a}$ & $\mathrm{n} / \mathrm{a}$ & $\mathrm{n} / \mathrm{a}$ & $\mathrm{n} / \mathrm{a}$ & $\mathrm{n} / \mathrm{a}$ & Sommen et al., 2016 [11] \\
\hline c. $4903 \mathrm{G}>\mathrm{A}$ & p.E1635K & $\mathrm{n} / \mathrm{a}$ & $\mathrm{n} / \mathrm{a}$ & $\mathrm{n} / \mathrm{a}$ & $\mathrm{n} / \mathrm{a}$ & $\mathrm{n} / \mathrm{a}$ & $\mathrm{n} / \mathrm{a}$ & $\mathrm{n} / \mathrm{a}$ & $\mathrm{n} / \mathrm{a}$ & $\mathrm{n} / \mathrm{a}$ & $\mathrm{n} / \mathrm{a}$ & Miyagawa et al., 2013 [16] \\
\hline c. $5176 \mathrm{C}>\mathrm{T}$ & p.R1726W & $\mathrm{n} / \mathrm{a}$ & $\mathrm{n} / \mathrm{a}$ & $\mathrm{n} / \mathrm{a}$ & $\mathrm{n} / \mathrm{a}$ & $\mathrm{n} / \mathrm{a}$ & $\mathrm{n} / \mathrm{a}$ & $\mathrm{n} / \mathrm{a}$ & $\mathrm{n} / \mathrm{a}$ & $\mathrm{n} / \mathrm{a}$ & $\mathrm{n} / \mathrm{a}$ & Seco et al., 2017 [22] \\
\hline \multirow{2}{*}{ c. $5384 \mathrm{G}>\mathrm{A}$} & p.R1795H & $\mathrm{n} / \mathrm{a}$ & $\mathrm{n} / \mathrm{a}$ & $\mathrm{n} / \mathrm{a}$ & $\mathrm{n} / \mathrm{a}$ & $\mathrm{n} / \mathrm{a}$ & $\mathrm{n} / \mathrm{a}$ & $\mathrm{n} / \mathrm{a}$ & $\mathrm{n} / \mathrm{a}$ & $\mathrm{n} / \mathrm{a}$ & $\mathrm{n} / \mathrm{a}$ & Moteki et al., 2017 [23] \\
\hline & & $\mathrm{M}$ & $30 \mathrm{~s}$ & $\mathrm{n} / \mathrm{a}$ & $51 \mathrm{Y}$ & Gently sloping & 56.25 & Moderate & $\mathrm{No}^{+}$ & $\mathrm{n} / \mathrm{a}$ & $\mathrm{n} / \mathrm{a}$ & \\
\hline \multirow[t]{2}{*}{ c. $5516 \mathrm{C}>\mathrm{A}$} & p.A1839D & $\mathrm{M}$ & $30 \mathrm{~s}$ & Yes & $45 \mathrm{Y}$ & Gently sloping & 50 & Moderate & $\mathrm{n} / \mathrm{a}^{+}$ & $\mathrm{n} / \mathrm{a}$ & $\mathrm{n} / \mathrm{a}$ & Wang et al., 2020 [24] \\
\hline & & $\mathrm{M}$ & $10 \mathrm{~s}$ & Yes & $29 \mathrm{Y}$ & Flat & 56.25 & Moderate & No $\ddagger$ & $\mathrm{n} / \mathrm{a}$ & $\mathrm{n} / \mathrm{a}$ & \\
\hline c. $5602 \mathrm{G}>\mathrm{A}$ & p.A1868T & $\mathrm{M}$ & $\mathrm{n} / \mathrm{a}$ & $\mathrm{n} / \mathrm{a}$ & $\mathrm{n} / \mathrm{a}$ & $\mathrm{n} / \mathrm{a}$ & 66.3 & $\mathrm{n} / \mathrm{a}$ & $\mathrm{n} / \mathrm{a}$ & $\mathrm{n} / \mathrm{a}$ & $\mathrm{n} / \mathrm{a}$ & Kim et al., 2016 [18] \\
\hline
\end{tabular}

${ }^{+}$Low-amplitude in oVEMP; $\ddagger$ labyrinth reactivity lower in caloric test; $\mathrm{n} / \mathrm{a}$, not available; $\mathrm{CI}$, Cochlear Implant. 
In this study, one nonsense variant, one frameshift variant, one non-frameshift variant causing a nonsense codon, six missense variants, and one variant with frameshift and missense variants in the cis allele were identified. One nonsense variant was located near the N-terminal region of MYH14, three missense variants were located in the Myosin head domain, three missense variants were located in the coiled-coil domain, and the other three variants were located in the C-terminal region (Table 2, Figure 2).

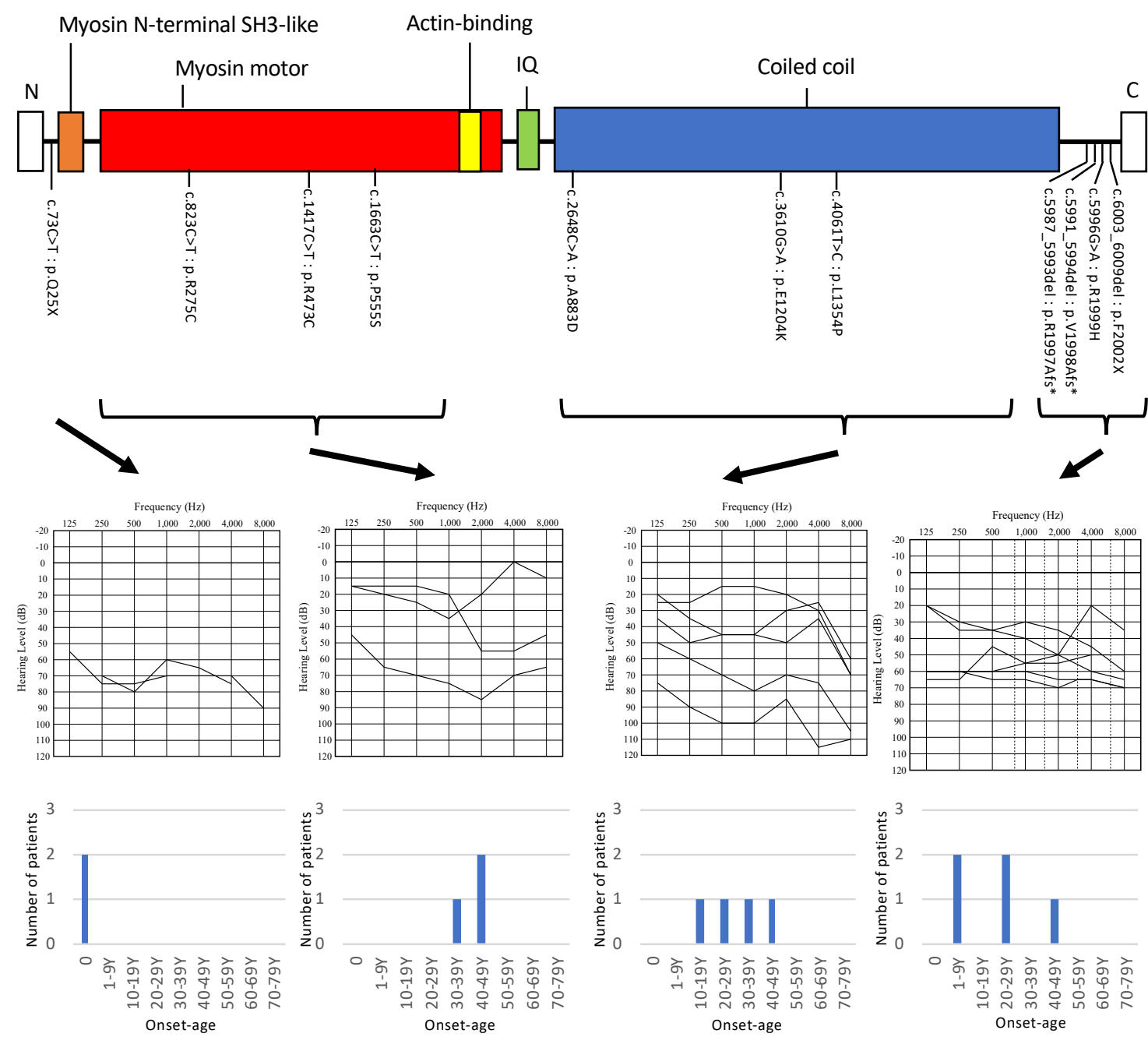

Figure 2. Domain structure of MYH14, identified variants, the overlapping audiograms from the better-hearing ear, and onset-age distribution for the patients with each domain variant. Domain structures are indicated based on UniProtKB (Q7Z406). Orange: Myosin N-terminal SH3 like domain, Red: Myosin motor domain, Yellow: Actin binding domain, Green: IQ domain, Blue: Coiled coil domain.

We included the 11 probands and six affected family members for clinical characteristics analysis. Regarding the onset of HL among the 11 probands and six family members identified, most of the patients showed late-onset hearing loss, and only two patients showed congenital onset (Figure 2). Both congenital HL patients had MYH14 variants located near the N-terminal region (Figure 2). The onset ages for the late-onset HL cases ranged from 3 to 44 (Table 1, Figure 2).

The audiometric configurations of the 11 probands and their affected family members were categorized into Flat $(n=11)$, Mid-frequency U-shaped $(n=2)$, High-frequency gently sloping $(n=1)$, High-frequency steeply sloping $(n=1)$, and Low-frequency ascending $(n=1)$ (Figure 1 , Table 1$)$. We could not identify any genotype-phenotype correlation among the domains (Figures 1 and 2). The severity of deafness also varied among cases. In the PTA calculated from the audiometric thresholds at four frequencies $(500,1000$, 
2000, and $4000 \mathrm{~Hz}$ ), 3 patients showed mild HL, 10 moderate HL, 2 severe HL, and 1 profound HL (Table 1). However, the one profound hearing loss case in Family No. 7 (Case I-4) had a history of repeated bilateral otitis media since childhood, and he had undergone bilateral middle ear surgery. After this middle ear surgery, he suffered bilateral deterioration in hearing. Thus, it is unclear whether the profound HL observed for this case was purely due to genetic causes. Among the 17 patients, at least 8 patients used hearing aids. Two of the eleven probands complained of dizziness, and one (Family No. 5) underwent an examination for nystagmus and caloric testing, but no obvious abnormal findings were observed.

Anamnestic evaluation of the 11 probands and 6 of their family members was also performed, and 12 were conscious of the progression of deafness (Table 1). Figure 3 shows serial audiograms for eight patients from six families. The audiograms for the betterhearing ear were used to evaluate progression. The patients who were observed for more than 10 years showed clear progression (Family No. 4 IV-2, Family No. 6 III-2, and Family No. 6 IV-2). Family No.9 IV-2 also showed hearing progression over two years. The observation periods for Family No. 7, No. 8, and No. 10 were too short to allow evaluation of progression (5 months to 16 months).
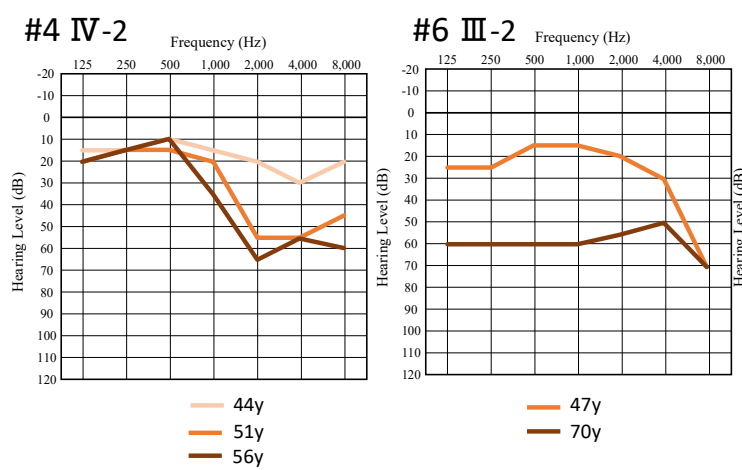

$$
\begin{array}{r}
-47 y \\
-70 y
\end{array}
$$

\#8 IV-2

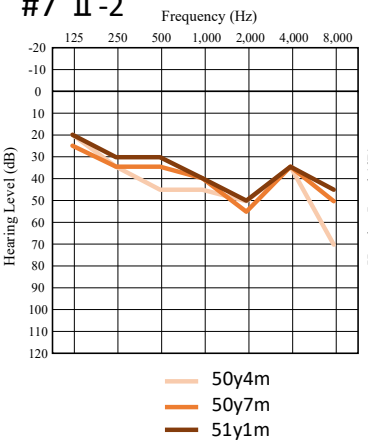

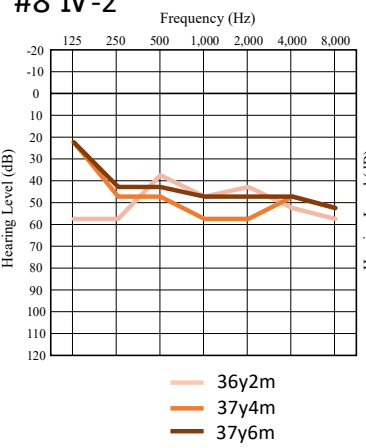

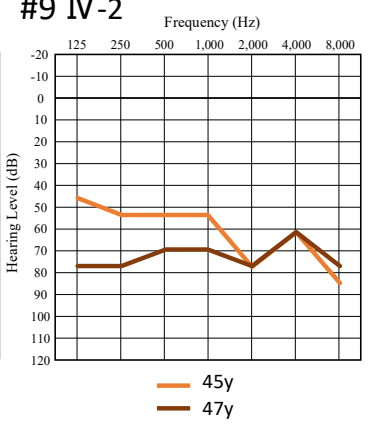

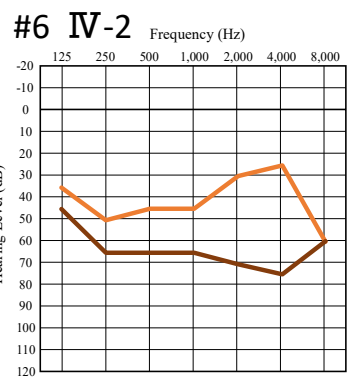

$$
\begin{array}{r}
-14 y \\
-37 y
\end{array}
$$
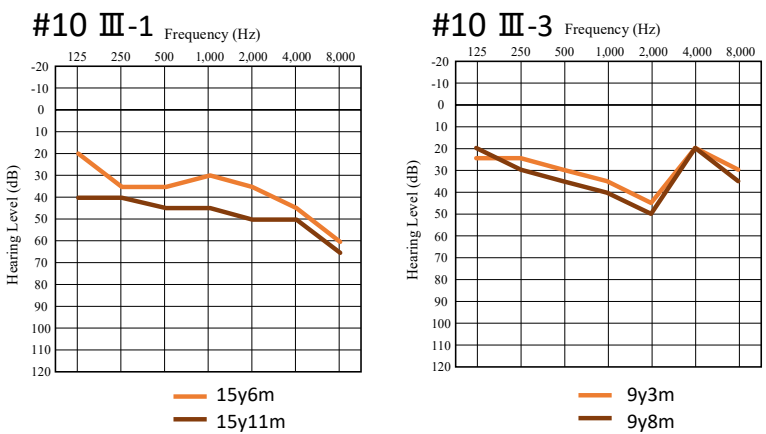

Figure 3. Serial audiograms of eight individuals from six families with MYH14 variants. 


\section{Discussion}

As MYH14-associated HL is rare, the currently available information regarding the variant spectrum and clinical characteristics is limited. In this study, using a cohort of $8074 \mathrm{HL}$ patients, we identified nine novel variants, and were able to summarize the variant spectrum. This is the largest cohort studied for MYH14-associated hearing loss to date. In addition, we were able to clarify the prevalence of MYH14 gene variants in patients; that is, $0.14 \%(11 / 8074)$ among HL patients and $0.82 \%$ among ADNSHL patients $(11 / 1336)$. To date, there have been four reports of the variant prevalence of MYH14-associated HL. Shearer et al. [7] reported that the frequency of MYH14-associated HL was 3.0\% among all HL patients in the USA ( $3 / 100$ probands). Sloan-Heggen et al. [8] reported that 5 of 1119 HL patients $(0.45 \%)$ carried MYH14 candidate pathogenic variants, and these variants accounted for 3.5\% of ADNSHL patients (5/141). Chen et al. [9] reported the prevalence of MYH14-associated HL in Chinese to be $2.59 \%$ (3/116). It is difficult to compare prevalence, as it depends on study subjects and pathogenicity classification methods. In this study, as stated in Section 2.2, more definitive criteria for pathogenicity were applied.

Various genes have been reported to be causative in ADNSHL families [6,8]; however, at present, there is no particular major responsible deafness gene for ADNSHL. Our screening using the same cohort clarified the frequencies for the other causative genes in ADNSHL patients; KCNQ4: 6.6\% [37], POU4F3: 4\% [38], TECTA: 2.9\% [39], WFS1: 2.5\% [40], MYO6: 2.4\% [41], ACTG1: 1.1\% [42], and EYA4: 0.9\% [43]. Although MYH14-associated $\mathrm{HL}$ is rare, the present results indicated that this gene should be included in HL screening, especially that for ADNSHL.

Among the 11 probands, two cases (Family No. 8 and 9) carried the same variant. These two cases were in unrelated families. As the mechanism of these commonly observed variants could have occurred by founder mutation or in a mutational hotspot, haplotype analysis could afford a method of clarifying their genesis. However, we obtained only the proband sample for Family No. 8, and could not perform haplotype analysis.

With regard to onset age, a majority of patients were shown to be have experienced adult onset occurring later than 20 years of age $(9 / 14,64.3 \%)$. The onset age had not been reported previously, except for two pedigrees with congenital or prelingual onset (Table 3). In this study, we clearly determined that late onset was one of the characteristic clinical features of MYH14-associated HL. It was noteworthy, however, that there were four patients with an onset age under 10 years old. Among them, HL in one patient (Family No. 1, III-2) was found through the newborn hearing screening program. It is interesting to note that this case carried the same nonsense variant (c.73C > T p.Q25X) as that previously reported in a congenital HL case ${ }^{4}$. This variant was located near the $\mathrm{N}$-terminal region. As all other variants located in the other domains identified in this study did not cause congenital HL, truncation within this domain may cause congenital HL through haploinsufficiency.

Information regarding the severities and audiometric configurations of MYH14associated HL in previous reports was also fragmentary (Table 3). The present study showed that a majority of patients exhibited moderate $(62.5 \%, 10 / 16)$ and Flat-type HL $(68.8 \%, 11 / 16)$ (Figure 1, Table 1).

In terms of the progression of MYH14-associated HL, $92.3 \%$ of patients $(12 / 13)$ had noticed the progression of their HL (Table 1). In this study, as shown in Figure 3, at least five patients showed progression based on serial audiometric evaluations. In particular, three patients (Family No. 4 IV-2, Family No. 6 III-2, and Family No. 6 IV-2) underwent serial audiometric evaluations over more than 10 years and showed obvious progression of HL. The present data strongly supported the notion that the progression of $\mathrm{HL}$, which has been previously reported [3,4], is one of the characteristic features of MYH14-associated HL.

With regards to intervention, 8 of the 16 patients used hearing aids (HAs) (Table 1), indicating that hearing aids should be recommended as hearing devices. In this study, no patient received cochlear implantation, suggesting that the HL was within the hearing range for which HAs are indicated in most cases. Liu et al. [15] reported a case in which 
cochlear implantation was performed for a patient with a MYH14 variant. However, it should be noted that this case also had a MYO15A compound heterozygous variant. Therefore, it is unclear whether the MYH14 variant itself causes profound hearing loss for which cochlear implantation is indicated.

We could not identify any genotype-phenotype correlations among the domains. In addition, we could not identify any genotype-phenotype correlations through combination of the clinical information obtained in our study and that of previously reported cases.

\section{Conclusions}

The present study revealed an updated variant spectrum and the clinical characteristics of MYH14-associated HL, including onset age, severity and progression of hearing loss, audiometric configuration, and recommended intervention. The information provided in this paper will play a crucial role in managing patients in the future.

Supplementary Materials: The following are available online at https:/ / www.mdpi.com/article/10 .3390 / genes12101623/s1, Table S1: The 68 deafness-causative genes analyzed in this study.

Author Contributions: Conceptualization, S.-i.K., S.-y.N. and S.-i.U.; methodology, S.-i.K., T.K. and S.-y.N.; software, S.-y.N.; validation, K.H. and T.K.; formal analysis, K.H. and S.-y.N.; investigation, S.-i.U.; data curation, K.H., S.-i.K., T.K., H.M. and S.-y.N.; writing—original draft preparation, K.H.; writing-review and editing, K.H., S.-i.K., T.K., H.M. and S.-y.N.; visualization, K.H.; supervision, S.-i.U.; project administration, S.-i.U.; funding acquisition, S.-i.U. All authors have read and agreed to the published version of the manuscript.

Funding: This study was funded by a Health and Labor Sciences Research Grant for Research on Rare and Intractable Diseases and Comprehensive Research on Disability Health and Welfare from the Ministry of Health, Labour and Welfare of Japan (S.U. H29-Nanchitou(Nan)-Ippan-031, 20FC1048); a Grant-in-Aid from the Japan Agency for Medical Research and Development (AMED) (S.U. 16kk0205010h0001, 17kk0205010h0002, 18kk0205010h0003, 16ek0109114h0002, 17ek0109114h0003, 18ek0109363h0001, 19ek0109363h0002, 20ek0109363h0003); and a Grant-in-Aid for Scientific Research (A) from the Ministry of Education, Culture, Sports, Science and Technology of Japan (S.U. 15H02565).

Institutional Review Board Statement: The study was conducted according to the guidelines of the Declaration of Helsinki, and approved by the Institutional Ethics Committee of Shinshu University School of Medicine (No. 387-4 September 2012, and No. 576-2 May 2017).

Informed Consent Statement: Informed consent was obtained from all patients (or from their next of kin, caretaker, or legal guardian in the cases of minors or children).

Data Availability Statement: The sequencing data are available in the DDBJ databank of Japan (Accession number: JGAS000323).

Acknowledgments: We thank Chihiro Morimoto, Kozo Kumakawa, Satoko Abe, Takahisa Abe, Hiromitsu Miyazaki, Kyoko Nagai, Satoshi Iwasaki, Kiyoshi Oda, Tomoko Shintani, and Takeshi Ishino for providing samples and clinical information. We also thank Sachiko Matsuda and Fumiko Tomioka for their technical assistance with this research.

Conflicts of Interest: The authors declare no conflict of interest. The funders had no role in the design of the study; in the collection, analyses, or interpretation of data; in the writing of the manuscript; or in the decision to publish the results.

\section{References}

1. Hereditary Hearing Loss Homepage. Available online: http:/ /hereditaryhearingloss.org/ (accessed on 30 December 2019).

2. Smith, R.J.H.; Bale, J.F.; White, K.R. Sensorineural hearing loss in children. Lancet 2005, 365, 879-890. [CrossRef]

3. Donaudy, F.; Snoeckx, R.; Pfister, M.; Zenner, H.P.; Blin, N.; Di Stazio, M.; Ferrara, A.; Lanzara, C.; Ficarella, R.; Declau, F.; et al. Nonmuscle myosin heavy-chain gene MYH14 is expressed in cochlea and mutated in patients affected by autosomal dominant hearing impairment (DFNA4). Am. J. Hum. Genet. 2004, 74, 770-776. [CrossRef] [PubMed]

4. Kim, B.J.; Kim, A.R.; Han, J.H.; Lee, C.; Oh, D.Y.; Choi, B.Y. Discovery of MYH14 as an important and unique deafness gene causing prelingually severe autosomal dominant nonsyndromic hearing loss. J. Gene Med. 2017, 4, 19. [CrossRef] [PubMed]

5. Yang, T.; Pfister, M.; Blin, N.; Zenner, H.P.; Pusch, C.M.; Smith, R.J. Genetic heterogeneity of deafness phenotypes linked to DFNA4. Am. J. Med. Genet. A 2013, 139, 9-12. [CrossRef] [PubMed] 
6. Iwasa, Y.I.; Nishio, S.Y.; Usami, S.I. Comprehensive Genetic Analysis of Japanese Autosomal Dominant Sensorineural Hearing Loss Patients. PLoS ONE 2016, 11, e0166781. [CrossRef]

7. Shearer, A.E.; Black-Ziegelbein, E.A.; Hildebrand, M.S.; Eppsteiner, R.W.; Ravi, H.; Joshi, S.; Guiffre, A.C.; Sloan, C.M.; Happe, S.; Howard, S.D.; et al. Advancing genetic testing for deafness with genomic technology. J. Med. Genet. 2013, 50, 627-634. [CrossRef]

8. Sloan-Heggen, C.M.; Bierer, A.O.; Shearer, A.E.; Kolbe, D.L.; Nishimura, C.J.; Frees, K.L.; Ephraim, S.S.; Shibata, S.B.; Booth, K.T.; Campbell, C.A.; et al. Comprehensive genetic testing in the clinical evaluation of 1119 patients with hearing loss. Hum. Genet. 2016, 135, 441-450. [CrossRef]

9. Chen, S.; Dong, C.; Wang, Q.; Zhong, Z.; Qi, Y.; Ke, X.; Liu, Y. Targeted Next-Generation Sequencing Successfully Detects Causative Genes in Chinese Patients with Hereditary Hearing Loss. Genet. Test. Mol. Biomark. 2016, 20, 660-665. [CrossRef]

10. Qing, J.; Yan, D.; Zhou, Y.; Liu, Q.; Wu, W.; Xiao, Z.; Liu, Y.; Liu, J.; Du, L.; Xie, D.; et al. Whole-Exome Sequencing to Decipher the Genetic Heterogeneity of Hearing Loss in a Chinese Family with Deaf by Deaf Mating. PLoS ONE 2014, 9, e109178. [CrossRef]

11. Sommen, M.; Schrauwen, I.; Vandeweyer, G.; Boeckx, N.; Corneveaux, J.J.; Ende, J.V.D.; Boudewyns, A.; Leenheer, E.D.; Janssens, S.; Claes, K.; et al. DNA Diagnostics of Hereditary Hearing Loss: A Targeted Resequencing Approach Combined with a Mutation Classification System. Hum. Mutat. 2016, 37, 812-819. [CrossRef]

12. Shearer, A.E.; Eppsteiner, R.W.; Booth, K.T.; Ephraim, S.S.; Gurrola, J., 2nd; Simpson, A.; Black-Ziegelbein, E.A.; Joshi, S.; Ravi, H.; Giuffre, A.C.; et al. Utilizing Ethnic-Specific Differences in Minor Allele Frequency to Recategorize Reported Pathogenic Deafness Variants. Am. J. Hum. Genet. 2014, 95, 445-453. [CrossRef] [PubMed]

13. Abouelhoda, M.; Faquih, T.; El-kalioby, M.; Alkuraya, F.S. Revisiting the morbid genome of Mendelian disorders. Genome Biol. 2016, 17, 235. [CrossRef] [PubMed]

14. Kim, N.K.D.; Kim, A.R.; Park, K.T.; Kim, S.Y.; Kim, M.Y.; Nam, J.-Y.; Woo, S.J.; Oh, S.-H.; Park, W.-Y. Whole-exome sequencing reveals diverse modes of inheritance in sporadic mild to moderate sensorineural hearing loss in a pediatric population. Genet. Med. 2015, 17, 901-911. [CrossRef]

15. Liu, W.-H.; Chang, P.-Y.; Chang, S.-C.; Lu, J.-J.; Wu, C.-M. Mutation screening in non-syndromic hearing loss patients with cochlear implantation by massive parallel sequencing in Taiwan. PLoS ONE 2019, 14, e0211261. [CrossRef] [PubMed]

16. Miyagawa, M.; Nishio, S.; Ikeda, T.; Fukushima, K.; Usami, S. Massively Parallel DNA Sequencing Successfully Identifies New Causative Mutations in Deafness Genes in Patients with Cochlear Implantation and EAS. PLoS ONE 2013, 8, e75793.

17. Choi, B.O.; Hee Kang, S.; Hyun, Y.S.; Kanwal, S.; Park, S.W.; Koo, H.; Kim, S.B.; Choi, Y.C.; Yoo, J.H.; Kim, J.W.; et al. A complex phenotype of peripheral neuropathy, myopathy, hoarseness, and hearing loss is linked to an autosomal dominant mutation in MYH14. Hum. Mutat. 2011, 32, 669-677. [CrossRef]

18. Kim, S.J.; Lee, S.; Park, H.J.; Kang, T.H.; Sagong, B.; Baek, J.I.; Oh, S.K.; Choi, J.Y.; Lee, K.Y.; Kim, U.K. Genetic association of MYH genes with hereditary hearing loss in Korea. Gene 2016, 591, 177-182. [CrossRef]

19. Vona, B.; Müller, T.; Nanda, I.; Neuner, C.; Hofrichter, A.H.H.; Schröder, J.; Bartsch, O.; Läßig, A.; Keilmann, A.; Schraven, S.; et al. Open Targeted next-generation sequencing of deafness genes in hearing-impaired individuals uncovers informative mutations Genet. Med. 2014, 16, 945-953. [CrossRef]

20. Iyadurai, S.; Arnold, W.D.; Kissel, J.T.; Ruhno, C.; McGovern, V.L.; Snyder, P.J.; Prior, T.W.; Roggenbuck, J.; Burghes, A.H.; Kolb, S.J. Variable phenotypic expression and onset in MYH14 distal HMN phenotype in a large, multigenerational North American family. Muscle Nerve 2017, 176, 139-148.

21. Almutawa, W.; Smith, C.; Sabouny, R.; Smit, R.B.; Zhao, T.; Wong, R.; Lee-Glover, L.; Desrochers-Goyette, J.; Ilamathi, H.S.; Suchowersky, O.; et al. The R941L mutation in MYH14 disrupts mitochondrial fission and associates with peripheral neuropathy. EBiolMedicine 2019, 45, 379-392. [CrossRef]

22. Seco, C.Z.; Wesdorp, M.; Feenstra, I.; Pfundt, R.; Hehir-Kwa, J.Y.; Lelieveld, S.H.; Castelein, S.; Gilissen, C.; Wijs, I.W.; Admiraal, R.J.; et al. The diagnostic yield of whole-exome sequencing targeting a gene panel for hearing impairment in the Netherlands. Eur. J. Hum. Genet. 2017, 25, 308-314. [CrossRef]

23. Moteki, H.; Azaiez, H.; Booth, K.T.; Shearer, A.E.; Sloan, C.M.; Kolbe, D.L.; Nishio, S.Y.; Hattori, M.; Usami, S.I.; Smith, R.J. Comprehensive genetic testing with ethnic-specific filtering by allele frequency in a Japanese hearing-loss population. Clin. Genet. 2017, 89, 466-472. [CrossRef]

24. Wang, M.; Zhou, Y.; Zhang, F.; Fan, Z.; Bai, X.; Wang, H. A novel MYH14 mutation in a Chinese family with autosomal dominant nonsyndromic hearing loss. BMC Med. Genet. 2020, 21, 154. [CrossRef]

25. Maekawa, K.; Nishio, S.; Abe, S.; Goto, S.I.; Honkura, Y.; Iwasaki, S.; Kanda, Y.; Kobayashi, Y.; Oka, S.; Okami, M.; et al. Mutational Spectrum and Clinical Features of Patients with LOXHD1 Variants Identified in an 8074 Hearing Loss Patient Cohort. Genes 2019, 10, 735. [CrossRef]

26. The Exome Aggregation Consortium Database (ExAC). Available online: http://exac.broadinstitute.org/ (accessed on 1 July 2019).

27. ToMMo 3.5KJPN-Integrative Japanese Genome Variation Database. Available online: https://jmorp.megabank.tohoku.ac.jp/20 $1905 /$ (accessed on 1 July 2019).

28. Wang, K.; Li, M.; Hakonarson, H. ANNOVAR: Functional annotation of genetic variants from high-throughput sequencing data. Nucleic Acids Res. 2010, 38, e164. [CrossRef] [PubMed]

29. Kumar, P.; Henikoff, S.; Ng, P.C. Predicting the effects of coding non-synonymous variants on protein function using the SIFT algorithm. Nat. Protoc. 2009, 4, 1073-1081. [CrossRef] [PubMed] 
30. Adzhubei, I.A.; Schmidt, S.; Peshkin, L.; Ramensky, V.E.; Gerasimova, A.; Bork, P.; Kondrashov, A.S.; Sunyaev, S.R. A method and server for predicting damaging missense mutations. Nat. Methods 2010, 7, 248-249. [CrossRef] [PubMed]

31. Schwarz, J.M.; Rodelsperger, C.; Schuelke, M.; Seelow, D. MutationTaster evaluates disease-causing potential of sequence alterations. Nat. Methods 2010, 7, 575-576. [CrossRef] [PubMed]

32. Reva, B.; Antipin, Y.; Sander, C. Predicting the functional impact of protein mutations: Application to cancer genomics. Nucleic Acids Res. 2011, 39, e118. [CrossRef] [PubMed]

33. Shihab, H.A.; Gough, J.; Cooper, D.N.; Stenson, P.D.; Barker, G.L.; Edwards, K.J.; Day, I.N.M.; Gaunt, T.R. Predicting the functional, molecular, and phenotypic consequences of amino acid substitutions using hidden Markov models. Hum. Mutat. 2013, $34,57-65$. [CrossRef]

34. Kircher, M.; Witten, D.M.; Jain, P.; O’Roak, B.J.; Cooper, G.M.; Shendure, J. A general framework for estimating the relative pathogenicity of human genetic variants. Nat. Genet. 2014, 46, 310-315. [CrossRef]

35. Richards, S.; Aziz, N.; Bale, S.; Bick, D.; Das, S.; Gastier-Foster, J.; Grody, W.W.; Hegde, M.; Lyon, E.; Spector, E.; et al. Standards and guidelines for the interpretation of sequence variants: A joint consensus recommendation of the American College of Medical Genetics and Genomics and the Association for Molecular Pathology. Genet. Med. 2015, 17, 405-424. [CrossRef]

36. Mazzoli, M.; Camp, G.V.; Newton, V.; Giarbini, N.; Declau, F.; Parving, A. Recommendations for the Description of Genetic and Audiological Data for Families with Nonsyndromic Hereditary Hearing Impairment. Audiol. Med. 2003, 1, 148-150.

37. Naito, T.; Nishio, S.; Iwasa, Y.; Yano, T.; Kumakawa, K.; Abe, S.; Ishikawa, K.; Kojima, H.; Namba, A.; Oshikawa, C.; et al. Comprehensive Genetic Screening of KCNQ4 in a Large Autosomal Dominant Nonsyndromic Hearing Loss Cohort: GenotypePhenotype Correlations and a Founder Mutation. PLoS ONE 2013, 8, e63231. [CrossRef]

38. Kitano, T.; Miyagawa, M.; Nishio, S.; Moteki, H.; Oda, K.; Ohyama, K.; Miyazaki, H.; Hidaka, H.; Nakamura, K.; Murata, T.; et al. POU4F3 mutation screening in Japanese hearing loss patients: Massively parallel DNA sequencing-34based analysis identified novel variants associated with autosomal dominant hearing loss. PLoS ONE 2017, 12, e0177636. [CrossRef] [PubMed]

39. Yasukawa, R.; Moteki, H.; Nishio, S.; Ishikawa, K.; Abe, S.; Honkura, Y.; Misako Hyogo, M.; Mihashi, R.; Ikezono, T.; Shintani, T.; et al. The prevalence and clinical characteristics of TECTA-associated autosomal dominant hearing loss. Genes 2019, $10,744$. [CrossRef]

40. Kobayashi, M.; Miyagawa, M.; Nishio, S.; Moteki, H. WFS1 mutation screening in a large series of Japanese hearing loss patients: Massively parallel DNA sequencing-based analysis. PLoS ONE 2018, 13, e0193359. [CrossRef] [PubMed]

41. Oka, S.; Day, T.F.; Nishio, S.; Moteki, H.; Miyagawa, M.; Morita, S.; Izumi, S.; Ikezono, T.; Abe, S.; Nakayama, J.; et al. Clinical characteristics and in vitro analysis of MYO6 variants causing late-onset progressive hearing loss. Genes 2020, 11, 273. [CrossRef]

42. Miyajima, H.; Moteki, H.; Day, T.; Nishio, S.; Murata, T.; Ikezono, T.; Takeda, H.; Abe, S.; Iwasaki, S.; Takahashi, M.; et al. Novel ACTG1 mutations in patients identified by massively parallel DNA sequencing cause progressive hearing loss. Sci. Rep. 2020, 10, 7056. [CrossRef]

43. Shinagawa, J.; Moteki, H.; Nishio, S.; Ohyama, K.; Otsuki, K.; Iwasaki, S.; Masuda, S.; Oshikawa, C.; Ohta, Y.; Arai, Y.; et al. Prevalence and clinical features of hearing loss caused by EYA4 variants. Sci. Rep. 2020, 10, 3662. [CrossRef] 